\title{
Secondary and tertiary ovarian cancer recurrence: what is the best management?
}

\author{
Simone Garzon ${ }^{1 \wedge}$, Antonio Simone Laganà ${ }^{\wedge}$, Jvan Casarin ${ }^{1} \wedge$, Ricciarda Raffaelli $^{2} \wedge$, Antonella Cromi ${ }^{1 \wedge}$, \\ Massimo Franchi $^{2} \wedge$, Fabio Barra ${ }^{3,4} \wedge$, Ibrahim Alkatout ${ }^{5} \wedge$, Simone Ferrero ${ }^{3,4} \wedge$, Fabio Ghezzi ${ }^{1 \wedge}$ \\ ${ }^{1}$ Department of Obstetrics and Gynecology, "Filippo Del Ponte” Hospital, University of Insubria, Varese, Italy; ${ }^{2}$ Department of Obstetrics and \\ Gynecology, AOUI Verona, University of Verona, Verona, Italy; ${ }^{3}$ Academic Unit of Obstetrics and Gynecology, Ospedale Policlinico San Martino, \\ Genoa, Italy; ${ }^{4}$ Department of Neurosciences, Rehabilitation, Ophthalmology, Genetics, Maternal and Child Health (DiNOGMI), University \\ of Genoa, Genoa, Italy; ${ }^{5}$ Kiel School of Gynecological Endoscopy, Department of Gynecology and Obstetrics, University Hospitals Schleswig- \\ Holstein, Kiel, Germany \\ Contributions: (I) Conception and design: S Garzon, AS Laganà, J Casarin, F Barra; (II) Administrative support: M Franchi, S Ferrero, F Ghezzi; \\ (III) Provision of study materials or patients: None; (IV) Collection and assembly of data: None; (V) Data analysis and interpretation: R Raffaelli, A \\ Cromi, I Alkatout; (VI) Manuscript writing: All authors; (VII) Final approval of manuscript: All authors. \\ Correspondence to: Antonio Simone Laganà, MD, PhD. Department of Obstetrics and Gynecology, "Filippo Del Ponte" Hospital, University of \\ Insubria, Piazza Biroldi 1, 21100, Varese, Italy. Email: antoniosimone.lagana@uninsubria.it.
}

\begin{abstract}
Ovarian cancer with complete clinical response recurs with a high rate. Recurrence is observed in almost $25 \%$ of cases with early-stage diseases and in more than $80 \%$ with more advance stages. Based on a platinum-free interval cut-off of 6 months, the first recurrence is usually classified in platinum-sensitive versus platinum-resistant, reflecting the biological characteristics underlying the clinical behavior. After this first recurrence, the patients are rarely cured, but second-line therapy can provide significant clinical responses, particularly in first platinum-sensitive recurrence. The approach to secondary and tertiary recurrence follows the same general principles applied in the first recurrence. Platinum-sensitivity based on the treatment-free interval defines the available chemotherapeutic regimens, whit less therapeutic options and a generally worse prognosis in platinum-resistant recurrent disease. Nevertheless, in this scenario, the introduction of new targeted therapies changed the prognosis of patients with both platinum-sensitive and platinum-resistant recurrence. The first introduced antiangiogenic therapy resulted able to improve prognosis in recurrent disease both as a single-agent and combined therapy, although the growing adoption in the first line therapy requires further investigation to prove their efficacy after repeated use. More recently, the approach to secondary, tertiary, and later recurrence has been changed by the introduction of PARP inhibitors, which resulted effective as maintenance monotherapy in both platinum-sensitive and platinumresistant recurrence when the genetic background of the tumor allows their application with a significant improvement of oncological outcomes. Overall, although the growing body of promising therapeutic options to approach recurrent ovarian cancer, all the available evidence suggests that the best unique management of secondary and tertiary recurrence does not exist but should be personalized based on the disease characteristics, previous treatments, patient characteristics, and patient preference. On that basis, in this review, we report a general and complete overview of the approach at the secondary and tertiary ovarian cancer recurrence with the aim to provide a wide vision on the multiple available therapeutic options.
\end{abstract}

Keywords: Ovarian cancer; recurrence; platinum-based chemotherapy; bevacizumab; PARP inhibitors

\footnotetext{
^Simone Garzon: ORCID: 0000-0002-5840-699X; Antonio Simone Laganà: ORCID: 0000-0003-1543-2802; Jvan Casarin: ORCID: 00000001-9519-1097; Ricciarda Raffaelli: ORCID: 0000-0001-6162-4114; Antonella Cromi: ORCID: 0000-0003-1697-5139; Massimo Franchi: ORCID: 0000-0001-6156-158X; Fabio Barra: ORCID: 0000-0003-4117-6603; Ibrahim Alkatout: ORCID: 0000-0002-7194-6034; Simone Ferrero: ORCID: 0000-0003-2225-5568; Fabio Ghezzi: ORCID: 0000-0003-3949-5410
} 
Submitted Mar 09, 2020. Accepted for publication Apr 25, 2020.

doi: 10.21037 /gs-20-325

View this article at: http://dx.doi.org/10.21037/gs-20-325

\section{Introduction}

Ovarian cancer is the fifth cancer per death in women and the first among gynecological cancer in western countries (1). Among malignant ovarian neoplasms, epithelial ovarian cancers are more than $90 \%$ of cases (2). The 5 -year survival is lower than $50 \%$, with a median age at diagnosis of 63 years, and more than $70 \%$ of patients who present with advanced disease (1). Epithelial ovarian cancer is a heterogeneous group of cancer, including four main categories (serous, endometrioid, mucinous, clear cell) and two main grades (low and high grade) with different molecular characteristics. About $70 \%$ of diagnosis involves serous histology that, in the majority of cases, is of high grade (2-5). Although treatment recommendation may be different between high- and low-grade diseases, the general principles are similar, with the relative resistance to standard chemotherapy regimens being a characteristic of low-grade tumors $(4,6,7)$.

In general, after the first-line therapy, including appropriate staging and debulking surgery followed by adjuvant platinum-based chemotherapy $(6,8-11)$, ovarian cancer with complete clinical response recurs with a high rate (12-14). Recurrence is observed in almost $25 \%$ of cases with early-stage diseases and in more than $80 \%$ with more advances stages (14-16). For the management of the first recurrence after complete remission of the disease, it has been standard for the clinical practice the classification of recurrence in platinum-sensitive versus platinum-resistant/refractory disease, based on the cutoff of 6 months after completing chemotherapy (17). The platinum-free interval of 6 months is an arbitrary cutoff in the classification of recurrences aimed to reflect the biological characteristics underlying the clinical behavior of the disease, which are intrinsically related to the efficacy of the treatment adopted in the previous line of therapy (17-19). Traditionally, the clinical classification of the first recurrence guides the choice of the most appropriate treatment among different options. Disease relapse before six months after completing chemotherapy suggests a different chemotherapeutic regimen, the inclusion in clinical trials, or the adoption of palliative and supportive care $(6,20)$. Conversely, the management of first recurrences over six months after chemotherapy completion is based on the combined platinum-based chemotherapy used in the first line of treatment with the option to consider a secondary cytoreductive surgery $(6,17,20-22)$. However, with the introduction of anti-angiogenesis therapies and of poly-adenosine diphosphate [ADP]-ribose polymerases (PARP) inhibitors, the approach to recurrent disease is going to change both in the classification and in treatment, particularly for the platinum-sensitive disease (23).

After the first ovarian cancer recurrence, the patients are rarely cured, but second-line therapy can provide significant clinical responses (14). In case of further recurrence, the same principles adopted in the treatment of the first recurrence guide the management of the second, third, and later recurrences, with the choice of the third/fourth line therapy based on previous first and second lines (6). Moreover, performance status, organ function, residual toxicity, and the risk of hypersensitivity reaction need to be considered before repeat treatments $(24,25)$. Particularly in the case of multiple courses of therapy, the patients can experience excessive toxicity and may require lower doses (6). Regardless of the selected treatment regimen for the recurrence, re-evaluation of patients after 2-4 cycles is recommended to assess the disease response. After two previous lines of consecutive therapeutic regimens, patients with platinum-resistant/refractory disease without evidence of clinical benefit are less likely to benefit from additional therapy (26). In general, a single best management of secondary and tertiary recurrence does not exist but should be personalized based on the disease characteristics (treatment-free interval, genetic profile), previous treatments, patient characteristics (performance status, previous toxicity), and patient preference (6,27-31). The recently introduced anti-angiogenesis therapies and PARP inhibitors should always be considered in eligible patients with secondary or further recurrence if previously not adopted (6).

\section{Secondary and tertiary platinum-sensitive recurrence}

\section{Platinum-based chemotherapy}

Disease relapse after the first-line platinum-based chemotherapy regimen at 6 months or later defines 
platinum-sensitive recurrent disease (17). In these patients, six cycles of platinum-based combined chemotherapy regimen is the recommended second-line therapy, and the single agent carboplatin or cisplatin is considered if the combined regimen is not tolerated although less effective $(17,21,22,32-40)$. In this population, a complete response to second-line chemotherapy is reported ranging between $15-30 \%$ of patients, with an overall response between $30-70 \%$ that seems to improve with longer platinum-free interval $(17,23)$. In case of secondary or further recurrence after six months or more, the platinum-sensitive state can be considered still present, and platinum-based combined chemotherapy regimen can be adopted and are typically offered with a progressively shorter progression-free survival after each line of therapy (41). Therefore, the probability that the subsequent recurrence is still platinumsensitive reduces, with subsequent reduced use of platinumbased regimens. Platinum sensitivity was reported as an independent predictor of progression-free survival until the third recurrence, and treatment of recurrence was reported as an independent predictor of overall-survival up to the fifth recurrence (41).

\section{Cytoreductive surgery}

In case of recurrence after a disease-free interval of at least 6 months, second cytoreductive surgery can be considered an option in selected patients with the aim to achieve a complete resection $(42,43)$. Overall survival was reported increased proportionally to the resection rate, particularly in the case of residual disease $\leq 1 \mathrm{~cm}$ (44). Based on nonrandomized controlled studies, the second cytoreductive surgery is able to significantly improve the overall survival in platinum-sensitive recurrence if complete cytoreduction is achieved (45). These observations were confirmed by the preliminary results of the DESKTOP III trial, which reported improved progression-free survival with delayed secondary chemotherapy after optimal cytoreduction as compared to second-line platinum-based chemotherapy (46). The progression-free survival resulted of 14 months without and of 19.6 months with surgery (hazard ratio: 0.66, 95\% CI: $0.52-0.83$ ), and the median time to subsequent therapy was of 21 months after surgery versus 13.9 months without (hazard ratio: 0.61, 95\% CI: 0.48-0.77) (46). Conversely, the preliminary result of GOG-213 did not show a benefit of secondary cytoreduction followed by chemotherapy as compared to chemotherapy only (47). The median progression-free survival was of 18.2 months after surgery versus 16.5 months without (hazard ratio: $0.88,95 \% \mathrm{CI}: 0.70-1.11$ ), and the median overall survival was of 53.6 months with versus 65.7 months without surgery (hazard ratio: $1.28,95 \%$ CI: $0.92-1.79$ ). Both data refer to the interim analysis of the two trials, in which the complete resection in the group underwent secondary surgery was achieved in a comparable proportion of women (67\% versus $63 \%)$. The main difference between the two trials is the adoption of bevacizumab based on the investigator's choice in the GOG-213 trial, which was adopted in a high proportion of women in both the secondary surgery and chemotherapy only arm, which may explain the observed differences with the DESKTOP III trial (47). Regardless of conflicting results, evidence suggests that secondary cytoreductive surgery should be considered particularly when complete cytoreduction is achievable. Similar to secondary surgery, observational studies suggest that complete tertiary cytoreductive surgery has a potentially positive effect on patient overall survival (48). Multicenter retrospective studies consistently reported the complete tertiary cytoreductive surgery as one of the most important independent predictors of longer overall survival, which was achieved in the $68-49 \%$ of patients (49-52). Noteworthy, initial ovarian cancer stage, site of recurrence, and timing of previous recurrences after treatments represent further prognostic factors suggesting a key role of appropriate patient selection (49-52). Evidence about quaternary cytoreductive surgery suggests a similar effect of complete cytoreduction in case of tertiary recurrence, with the treatment-free interval and the number and site of recurrence representing important predictive factors $(53,54)$. Based on available evidence, cytoreductive surgery is an option for the management of secondary and further recurrences in selected patients in who optimal cytoreduction is achievable and the treatment-free interval is enough to suggest a benefit provided by the surgery. Noteworthy, in this scenario, after the demonstrated role of hyperthermic intraoperative intraperitoneal chemoperfusion (HIPEC) in the first line of therapy $(6,55,56)$, a growing body of evidence suggests that the HIPEC may represent a complimentary, safety, and feasible additional treatment option in secondary and further recurrences, although further evidence is required (57-62).

\section{Antiangiogenic therapies}

Angiogenesis has a key role in ovarian cancer growth and metastasis and is driven by different growth factors, such as 
platelet-derived growth factor (PDGF), vascular endothelial growth factor (VEGF), fibroblast growth factor (FGF), and angiopoietin-1 and -2 (63). Among antiangiogenic therapies, bevacizumab has been the first drug approved for the treatment of recurrent ovarian cancer by the FDA in 2014. Bevacizumab is a humanized anti-VEGF monoclonal antibody that was reported able to improve progression-free survival and overall survival as a single agent in recurrent ovarian cancer after multiple lines of therapy regardless of the platinum-sensitivity state $(64,65)$. The use of bevacizumab for the treatment of recurrent platinum-sensitive ovarian cancer has been investigated in two randomized phase III trials (63). OCEAN trial investigated bevacizumab as an addition to gemcitabine and carboplatin versus gemcitabine and carboplatin alone as second-line chemotherapy for first platinum-sensitive recurrence. Gemcitabine and carboplatin plus bevacizumab followed by bevacizumab until progression was associated with a improve progression-free survival (66). Similarly, GOG-213 trial reported improved progression-free survival in the group who received carboplatin and paclitaxel with bevacizumab followed by maintenance (47). However, both trials failed to report a statistically significant improvement of overall survival, although the GOG-213 trial reported an absolute improvement of 5 -month that was not observed in the OCEAN trial $(47,66)$. This difference is supposed to be related to the backbone chemotherapy regimen, with carboplatin and paclitaxel considered better than gemcitabine and carboplatin as association with bevacizumab (63). Based on this evidence, bevacizumab is considered for the treatment of platinum-sensitive recurrence as single agent as well as in combination with gemcitabine and carboplatin or carboplatin and paclitaxel for the treatment of platinum-sensitive recurrence, particularly in patients with ascites, although it is not the first recommended option due to the absent proof of improved survival (6). Noteworthy, the cost and toxicity of bevacizumab, especially bowel perforation, renal dysfunction, and hypertension, combined with the absent improved overall survival provide some concerns about the cost-effectiveness (63).

All the reported evidence about the use of bevacizumab in platinum-sensitive recurrence is primarily based on the first recurrence, with limited evidence on the use in secondary or further platinum-sensitive recurrence primarily focused on the use of bevacizumab as a single agent (65). However, in the case of secondary or further platinumsensitive recurrence without previous administration, bevacizumab can be considered an option in the treatment of these patients as a single agent or in combination (6). Noteworthy, given the introduction of bevacizumab in the first-line therapy $(67,68)$, as well as the use of bevacizumab in first recurrence $(47,66)$, the use of a second of further line of bevacizumab in the following platinum-sensitive recurrence is still undefined, although preliminary data suggest improved progression-free survival (69).

Other antiangiogenic therapies have been investigated in recurrent platinum-sensitive ovarian cancer. The ICON6 trial investigated the additional and maintenance therapy of cediranib (VEGF and c-KIT inhibitor) added to platinumbased chemotherapy, reporting an improved progressionfree survival and overall survival (70-72). TRINOVA-1 trial investigated trebananib (angiopoietin-1 and -2 inhibitor) in platinum-sensitive and platinum-resistant recurrence. The progression-free survival resulted improved in the group of patients who underwent trebananib plus paclitaxel as compared to paclitaxel alone in the platinum-sensitive group only, with a benefit in terms of overall survival in the subgroup with ascites (73). Regardless of the available evidence, only in phase II trial secondary platinumsensitive recurrence are included in the study population, with limited evidence focusing on the administration of antiangiogenic therapy in secondary and further recurrence.

\section{PARP inhibitors}

PARP inhibitors represent the true promising therapeutic approach for secondary and tertiary platinum-sensitive recurrence in ovarian cancer. PARP inhibitors are selectively effective against any homologous recombination deficient (HRD) cells, such as cells caring biallelic BRCA1 or -2 deficiency (74). PARP enzymes are components of the nonhomologous DNA repair machinery, which through the base-excision pathway repair single-strand breaks. PARP inhibitors impede this low-fidelity repair, and the persistence of single-strand breaks evolves in doublestranded breaks that cannot be repaired in HRD cells with subsequent lethality (75). It is estimated that almost $50 \%$ of high-grade serous ovarian cancers are HRD (15\% germline BRCA mutation, $6 \%$ somatic BRCA mutation, $20 \%$ other components determining HRD) (76), which has been correlated to both platinum-sensitivity and responsiveness to PARP inhibitors $(77,78)$.

Olaparib is a PARP-1, -2 , and -3 inhibitor and has been the first PARP inhibitor to be investigated and approved for the treatment of ovarian cancer (6). Study 19 was a phase II 
trial that investigates Olaparib as maintenance monotherapy versus placebo in secondary platinum-sensitive recurrence or in disease with a partial response after the second line of platinum-based chemotherapy. The BRCA-mutated group reported the greatest benefit in terms of progressionfree survival and overall survival, although a benefit was observed regardless of BRCA state (79). SOLO2/ENGOTOv21 phase III trial focused on the same population with secondary platinum-sensitive recurrence or at least partial response to the second platinum-based chemotherapy line with known or suspected BRCA-mutation. The trial reported a significant improvement in progression-free survival, regardless of the administration of bevacizumab in previous therapeutic lines, in the group who received Olaparib as maintenance monotherapy versus the group who received a placebo (80).

Rucaparib is a PARP inhibitor similar to Olaparib that was reported effective and safety in phase I/II trial in patients with recurrent platinum-sensitive high-grade ovarian cancer and a germline BRCA mutation $(81,82)$. The ARIEL2 part1 trial demonstrated improved progression-free survival with Rucaparib monotherapy in patients with platinumsensitive recurrence who underwent at least one prior platinum-based regimen and who had germline or somatic BRCA mutation (hazard ratio: 0.27, 95\% CI: 0.16-0.44), or BRCA wild-type with HRD tumor (hazard ratio: 0.62, 95\% CI: $0.42-0.90$ ) showed by high loss of heterozygosis in tumor genomic (mutation or methylation of other genes involved in homologous recombination), as compared with women without loss of heterozygosity (83). The median progression-free survival was reported of 12.8 months in women with BRCA mutation, of 5.7 months in women with HRD tumor, and of 5.2 months in women without loss of heterozygosity (83). ARIEL3 trial confirmed the statistically significant improvement of progression-free survival in patients with platinum-sensitive recurrent ovarian cancer, with at least two previous lines of platinumbased chemotherapy and mutated BRCA (somatic or germinal) or HRD tumor (84).

Niraparib is a PARP-1 and PARP-2 inhibitor with a longer half-life, absent interaction with cytochrome $\mathrm{P} 450$ enzymes, and the ability to cross the blood-brain barrier. The ENGOT-OV16/NOVA trial investigated Niraparib in women with a germline BRCA mutation or high-grade serous ovarian cancers and secondary or further platinumsensitive recurrence or partial response after at least two or more previous line of platinum-based chemotherapy. In the case of partial response, the residual disease needed to be less than $2 \mathrm{~cm}$, and the progression-free survival was improved by Niraparib versus placebo in all the investigated subgroups: germline BRCA mutated group, HRD positive tumors, and HRD negative tumors. The magnitude of the improvement in progression-free survival was higher in the BRCA mutated group and lower in the HRD negative tumor group (85).

PARP inhibitors dramatically changed the prognosis of women with platinum-sensitive ovarian cancer. Based on the results of Study19/SOLO2 studying Olaparib, ARIEL3 investigating Rucaparib, and ENGOT-OV16/NOVA trial studying Niraparib the PARP inhibitors have been approved and are recommended as maintenance therapy in secondary or further platinum-sensitive recurrence of ovarian cancer or in case of partial response at the second or later platinum-based chemotherapy $(79,80,84-86)$. Although initially their administration was limited to BRCA mutated or HRD positive tumors, the results of NOVA trials reported Niraparib as effective in platinum-sensitive disease regardless of the BRCA or HRD status, which instead provides a prediction of response to treatment $(84,85)$.

Based on available evidence, Olaparib and Rucaparib have been approved as a single-agent therapy in patients with BRCA mutations or HRD positive tumors and recurrent platinum-sensitive or platinum-resistant ovarian cancer after 2 or more lines of chemotherapy (6). Conversely, Niraparib has been approved and recommended as maintenance monotherapy for platinum-sensitive recurrent disease or for disease with a partial response after the second or later line of platinum-based chemotherapy regardless of BRCA/HRD state (6).

Noteworthy, based on the potential synergistic effect of PARP inhibitors with platinum-based chemotherapy, being the PARP enzymes involved in the repair of the damage caused by chemotherapy, ongoing trials are investigating the combined approach in recurrent platinum-sensitive disease. A phase II trial on Olaparib plus carboplatin and paclitaxel in first or later platinum-sensitive recurrent disease versus chemotherapy alone has been the first to report a benefit provided by the combined therapy (87). Similarly, different ongoing trials are investigating PARP inhibitors combined with antiangiogenic agents (23).

PARP inhibitors are overall well tolerated with no difference in the quality of life as compared to placebo $(88,89)$. However, almost $10-15 \%$ of patients discontinue the therapy due to side effects that include myelosuppression, fatigue, nausea, and vomiting, although dose reduction is an option to maintain the therapy (90). 


\section{Secondary and tertiary platinum-resistant recurrence}

\section{Chemotherapy}

The platinum response is one of the major prognostic factors (91). Recurrent disease in less than 6 months after platinum-based chemotherapy is defined as platinumresistant recurrence and is usually associated with a poor prognosis. After two consecutive chemotherapy lines of treatment, patients with platinum-resistant/refractory disease without evidence of clinical response are less likely to benefit from additional therapy (26). Platinum-resistant recurrence can be evident after the first line of treatment as well as after more than one line of platinum-based chemotherapy and platinum-sensitive recurrence. In the case of initially platinum-sensitive disease, the probability that the subsequent recurrence is still platinum-sensitive reduces with the increase of chemotherapy lines, with subsequent reduced use of platinum-based regimens. As previously reported, platinum sensitivity was reported as an independent predictor of progression-free survival until the third recurrence, and treatment of recurrence was reported as independent predictor of overall-survival up to the fifth recurrence (41). Therefore, second, third, and later recurrences tend to became platinum-resistant. In these cases, salvage single-agent chemotherapy non-platinumbased is the first choice, being the multiagent chemotherapy associated with higher toxicity without improvement of oncological outcomes. Among different options, pegylated liposomal doxorubicin, paclitaxel, and topotecan are the most effective agents, although with limited benefit (6,92-98).

\section{Antiangiogenic therapies}

A significant improvement of oncological outcomes in platinum-resistant recurrence has been provided by bevacizumab. This humanized anti-VEGF monoclonal antibody has been reported able to improve progression-free survival and overall survival in phase II trial as a single agent in recurrent platinum-resistant disease after multiple lines of therapy $(64,65,99)$. Based on this evidence, bevacizumab is recommended as a targeted single-agent therapeutic option in platinum-resistant recurrent disease (6). Similar to OCEAN and GOG-213 trials for platinum-sensitive recurrence, combined therapy based on bevacizumab plus single chemotherapeutic agent for platinum-resistant recurrence has been investigated in the AURELIA phase III trial. Patients who received bevacizumab plus pegylated liposomal doxorubicin, paclitaxel, or topotecan reported a statistically significant improved progression-free survival as compared to chemotherapy only (100-102). Noteworthy, this trial included patients with only first or second platinum-resistant recurrence excluding patients with more than 2 previous lines of chemotherapy $(100,101)$. Therefore, similar to platinum-sensitive recurrence, available evidence on bevacizumab in platinum-resistant recurrence is primarily based on the first recurrence, with limited evidence on the use in secondary or further platinum-resistant recurrence, which is primarily focused on the use of bevacizumab as a single agent (65). However, in secondary, tertiary, or later platinum-resistant recurrence bevacizumab can be considered an option as a single agent as well as a combined therapy in eligible patients (6). Recent phase II trials further confirm the efficacy of a combination of single-agent chemotherapy and bevacizumab in platinum-resistant recurrence, regardless of the number of previous chemotherapy lines and previous administration of bevacizumab $(103,104)$, although further evidence about the repeat administration of bevacizumab in platinum-resistant recurrence is needed (105).

\section{PARP inbibitors}

PARP inhibitors represent an important therapeutic option in platinum-resistant recurrent disease given the limited therapeutic strategies. The ARIEL2 part2 trial investigated Rucaparib monotherapy including patients with platinumsensitive, -resistant, or -refractory recurrent high-grade ovarian cancer with somatic or germline BRCA mutation. Eligible patients were those who received three or four prior chemotherapy lines and a platinum-free interval of at least 6 months after the first-line chemotherapy. The integrated analysis limited to women with BRCA mutation of ARIEL2 part2 with ARIEL2 part 1 and Study 10 part 2A trials, reported response and better progression-free survival in the Rucaparib group regardless of the platinum state, but a lower effect has been reported in platinum-resistant/ refractory recurrent disease as compared to platinumsensitive recurrence. The progression-free survival was 11.1 (95\% CI: 7.3-12.8) months in platinum-sensitive recurrence versus 7.4 (95\% CI: 5.5-not reached) months and 5.3 (95\% CI: 1.7-not reached) months in platinumresistant and -refractory recurrence, respectively. Similarly, the response rate was $65.8 \%$ (95\% CI: $54.3-76.1 \%$ ) in platinum-sensitive versus $25.0 \%$ (95\% CI: $8.7-49.1 \%$ ) in platinum-resistant and $0 \%$ (95\% CI: $0-41.0 \%)$ in platinumrefractory recurrences. Noteworthy, the response rate 
to Rucaparib declines as the number of previous lines of treatment increases in all groups (82). Similar results have been reported for Olaparib with improved progressionfree survival in the treatment arm, although reporting lower response in platinum-resistant/refractory disease as compared to platinum-sensitive recurrence as well as a lower response rate with the increase of treatment lines number $(106,107)$. Based on this evidence, Olaparib and Rucaparib have been approved as a single-agent therapy in patients with BRCA mutations or HRD positive tumors and recurrent platinum-sensitive or platinum-resistant ovarian cancer after 2 or more lines of chemotherapy (6).

\section{Conclusion}

Only a limited proportion of patients with ovarian cancer develop a secondary, tertiary, or later recurrence and the evidence is overall limited. However, the general approach used in the first recurrence can guide the approach to subsequent recurrences. Platinum-sensitive secondary or tertiary recurrence allows the adoption of platinumbased chemotherapy, and in highly selected patients a further cytoreductive surgery can be considered. If not previously adopted, targeted therapy with bevacizumab can be an option, although from the secondary recurrence the introduction of PARP inhibitors in platinum-sensitive disease represents the true promising therapeutic option able to change the prognosis. In platinum-sensitive recurrence, the genetic investigation to identify BRCA mutated or HRD positive tumor is recommended if not already performed, although Niraparib has proven to be effective regardless genetic status. In secondary, tertiary, or later platinum-resistant disease the prognosis is completely different. Single-agent chemotherapy represents the usual practice, although the introduction of bevacizumab as single-agent or as combined therapy allows improving the prognosis. However, even in platinum-resistant recurrent disease, PARP inhibitors represent an important resource for patients with confirmed BRCA mutated or HRD positive tumors. Therefore, in platinum-resistant disease, the genetic investigation has a key role in the management of secondary and further recurrence. In conclusion, all the available evidence suggests that the best unique management of secondary and tertiary recurrence does not exist but should be personalized based on the disease characteristics, previous treatments, patient characteristics, and patient preference.

\section{Acknowledgments}

Funding: None.

\section{Footnote}

Provenance and Peer Review: This article was commissioned by the Guest Editor (Stefano Cianci) for the series "Ovarian Cancer Recurrence" published in Gland Surgery. The article was sent for external peer review organized by the Guest Editor and the editorial office.

Conflicts of Interest: All authors have completed the ICMJE uniform disclosure form (available at http://dx.doi. org/10.21037/gs-20-325). The series "Ovarian Cancer Recurrence" was commissioned by the editorial office without any funding or sponsorship. The authors have no other conflicts of interest to declare.

Ethical Statement: The authors are accountable for all aspects of the work in ensuring that questions related to the accuracy or integrity of any part of the work are appropriately investigated and resolved.

Open Access Statement: This is an Open Access article distributed in accordance with the Creative Commons Attribution-NonCommercial-NoDerivs 4.0 International License (CC BY-NC-ND 4.0), which permits the noncommercial replication and distribution of the article with the strict proviso that no changes or edits are made and the original work is properly cited (including links to both the formal publication through the relevant DOI and the license). See: https://creativecommons.org/licenses/by-nc-nd/4.0/.

\section{References}

1. Siegel RL, Miller KD, Jemal A. Cancer statistics, 2019. CA Cancer J Clin 2019;69:7-34.

2. Prat J. New insights into ovarian cancer pathology. Ann Oncol 2012;23 Suppl 10:x111-7.

3. Reade CJ, McVey RM, Tone AA, et al. The fallopian tube as the origin of high grade serous ovarian cancer: review of a paradigm shift. J Obstet Gynaecol Can 2014;36:133-40.

4. McCluggage WG. Morphological subtypes of ovarian carcinoma: a review with emphasis on new developments and pathogenesis. Pathology 2011;43:420-32.

5. Guadagno E, Pignatiello S, Borrelli G, et al. Ovarian borderline tumors, a subtype of neoplasm with 
controversial behavior. Role of Ki67 as a prognostic factor. Pathol Res Pract 2019;215:152633.

6. Armstrong DK, Alvarez RD, Bakkum-Gamez JN, et al. NCCN Guidelines Insights: Ovarian Cancer, Version 1.2019. J Natl Compr Canc Netw 2019;17:896-909.

7. Gourley C, Farley J, Provencher DM, et al. Gynecologic Cancer InterGroup (GCIG) consensus review for ovarian and primary peritoneal low-grade serous carcinomas. Int J Gynecol Cancer 2014;24:S9-13.

8. Gueli Alletti S, Capozzi VA, Rosati A, et al. Laparoscopy vs. laparotomy for advanced ovarian cancer: a systematic review of the literature. Minerva Med 2019;110:341-57.

9. Bellia A, Vitale SG, Lagana AS, et al. Feasibility and surgical outcomes of conventional and robot-assisted laparoscopy for early-stage ovarian cancer: a retrospective, multicenter analysis. Arch Gynecol Obstet 2016;294:615-22.

10. Casarin J, Lagana AS, Uccella S, et al. Surgical treatment of large adnexal masses: a retrospective analysis of 330 consecutive cases. Minim Invasive Ther Allied Technol 2019;2;1-9.

11. Giampaolino P, Della Corte L, Foreste V, et al. Unraveling a difficult diagnosis: the tricks for early recognition of ovarian cancer. Minerva Med 2019;110:279-91.

12. Nishino M, Jagannathan JP, Ramaiya NH, et al. Revised RECIST guideline version 1.1: What oncologists want to know and what radiologists need to know. AJR Am J Roentgenol 2010;195:281-9.

13. Eisenhauer EA, Therasse P, Bogaerts J, et al. New response evaluation criteria in solid tumours: revised RECIST guideline (version 1.1). Eur J Cancer 2009;45:228-47.

14. Salani R, Backes FJ, Fung MF, et al. Posttreatment surveillance and diagnosis of recurrence in women with gynecologic malignancies: Society of Gynecologic Oncologists recommendations. Am J Obstet Gynecol 2011;204:466-78.

15. Armstrong DK, Bundy B, Wenzel L, et al. Intraperitoneal cisplatin and paclitaxel in ovarian cancer. N Engl J Med 2006;354:34-43.

16. Trimbos JB, Parmar M, Vergote I, et al. International Collaborative Ovarian Neoplasm trial 1 and Adjuvant ChemoTherapy In Ovarian Neoplasm trial: two parallel randomized phase III trials of adjuvant chemotherapy in patients with early-stage ovarian carcinoma. J Natl Cancer Inst 2003;95:105-12.

17. Fung-Kee-Fung M, Oliver T, Elit L, et al. Optimal chemotherapy treatment for women with recurrent ovarian cancer. Curr Oncol 2007;14:195-208.

18. Lagana AS, Colonese F, Colonese E, et al. Cytogenetic analysis of epithelial ovarian cancer's stem cells: an overview on new diagnostic and therapeutic perspectives. Eur J Gynaecol Oncol 2015;36:495-505.

19. Lagana AS, Sofo V, Vitale SG, et al. Epithelial ovarian cancer inherent resistance: May the pleiotropic interaction between reduced immunosurveillance and drug-resistant cells play a key role? Gynecol Oncol Rep 2016;18:57-8.

20. Rodriguez-Freixinos V, Mackay HJ, Karakasis K, et al. Current and emerging treatment options in the management of advanced ovarian cancer. Expert Opin Pharmacother 2016;17:1063-76.

21. Parmar MK, Ledermann JA, Colombo N, et al. Paclitaxel plus platinum-based chemotherapy versus conventional platinum-based chemotherapy in women with relapsed ovarian cancer: the ICON4/AGO-OVAR-2.2 trial. Lancet 2003;361:2099-106.

22. Raja FA, Counsell N, Colombo N, et al. Platinum versus platinum-combination chemotherapy in platinum-sensitive recurrent ovarian cancer: a meta-analysis using individual patient data. Ann Oncol 2013;24:3028-34.

23. Mullen MM, Kuroki LM, Thaker PH. Novel treatment options in platinum-sensitive recurrent ovarian cancer: A review. Gynecol Oncol 2019;152:416-25.

24. Vitale SG, Capriglione S, Zito G, et al. Management of endometrial, ovarian and cervical cancer in the elderly: current approach to a challenging condition. Arch Gynecol Obstet 2019;299:299-315.

25. Cianci S, Rumolo V, Rosati A, et al. Sarcopenia in Ovarian Cancer Patients, Oncologic Outcomes Revealing the Importance of Clinical Nutrition: Review of Literature. Curr Pharm Des 2019;25:2480-90.

26. Griffiths RW, Zee YK, Evans S, et al. Outcomes after multiple lines of chemotherapy for platinum-resistant epithelial cancers of the ovary, peritoneum, and fallopian tube. Int J Gynecol Cancer 2011;21:58-65.

27. Cianci S, Tarascio M, Rosati A, et al. Sexual function and quality of life of patients affected by ovarian cancer. Minerva Med 2019;110:320-9.

28. Gueli Alletti S, Vizzielli G, Lafuenti L, et al. SingleInstitution Propensity-Matched Study to Evaluate the Psychological Effect of Minimally Invasive Interval Debulking Surgery Versus Standard Laparotomic Treatment: From Body to Mind and Back. J Minim Invasive Gynecol 2018;25:816-22.

29. Vitale SG, La Rosa VL, Rapisarda AM, et al. Comment on: "Anxiety and depression in patients with advanced ovarian cancer: a prospective study". J Psychosom Obstet Gynaecol 2017;38:83-4. 
30. Lagana AS, La Rosa VL, Rapisarda AM, et al. Psychological impact of fertility preservation techniques in women with gynaecological cancer. Ecancermedicalscience 2017;11:ed62.

31. Vitale SG, La Rosa VL, Rapisarda AMC, et al. Fertility preservation in women with gynaecologic cancer: the impact on quality of life and psychological well-being. Hum Fertil (Camb) 2018;21:35-8.

32. Rose PG. Gemcitabine reverses platinum resistance in platinum-resistant ovarian and peritoneal carcinoma. Int J Gynecol Cancer 2005;15 Suppl 1:18-22.

33. Pfisterer J, Plante M, Vergote I, et al. Gemcitabine plus carboplatin compared with carboplatin in patients with platinum-sensitive recurrent ovarian cancer: an intergroup trial of the AGO-OVAR, the NCIC CTG, and the EORTC GCG. J Clin Oncol 2006;24:4699-707.

34. Lawrie TA, Bryant A, Cameron A, et al. Pegylated liposomal doxorubicin for relapsed epithelial ovarian cancer. Cochrane Database Syst Rev 2013;(7):CD006910.

35. Staropoli N, Ciliberto D, Botta C, et al. Pegylated liposomal doxorubicin in the management of ovarian cancer: a systematic review and metaanalysis of randomized trials. Cancer Biol Ther 2014;15:707-20.

36. Benigno BB, Burrell MO, Daugherty P, et al. A phase II nonrandomized study of nab-paclitaxel plus carboplatin in patients with recurrent platinum-sensitive ovarian or primary peritoneal cancer. J Clin Oncol 2010;28:5011.

37. Gonzalez-Martin AJ, Calvo E, Bover I, et al. Randomized phase II trial of carboplatin versus paclitaxel and carboplatin in platinum-sensitive recurrent advanced ovarian carcinoma: a GEICO (Grupo Espanol de Investigacion en Cancer de Ovario) study. Ann Oncol 2005;16:749-55.

38. Cantu MG, Buda A, Parma G, et al. Randomized controlled trial of single-agent paclitaxel versus cyclophosphamide, doxorubicin, and cisplatin in patients with recurrent ovarian cancer who responded to first-line platinum-based regimens. J Clin Oncol 2002;20:1232-7.

39. Bolis G, Scarfone G, Giardina G, et al. Carboplatin alone vs carboplatin plus epidoxorubicin as second-line therapy for cisplatin- or carboplatin-sensitive ovarian cancer. Gynecol Oncol 2001;81:3-9.

40. Pujade-Lauraine E, Wagner U, Aavall-Lundqvist E, et al. Pegylated liposomal doxorubicin and carboplatin compared with paclitaxel and carboplatin for patients with platinum-sensitive ovarian cancer in late relapse. Springer Healthcare; 2013.

41. Hanker LC, Loibl S, Burchardi N, et al. The impact of second to sixth line therapy on survival of relapsed ovarian cancer after primary taxane/platinum-based therapy. Ann Oncol 2012;23:2605-12.

42. Harter P, Heitz F, Mahner S, et al. Surgical intervention in relapsed ovarian cancer is beneficial: pro. Ann Oncol 2013;24 Suppl 10:x33-4.

43. Gallotta V, Conte C, Giudice MT, et al. Secondary Laparoscopic Cytoreduction in Recurrent Ovarian Cancer: A Large, Single-Institution Experience. J Minim Invasive Gynecol 2018;25:644-50.

44. Bristow RE, Puri I, Chi DS. Cytoreductive surgery for recurrent ovarian cancer: a meta-analysis. Gynecol Oncol 2009;112:265-74.

45. Al Rawahi T, Lopes AD, Bristow RE, et al. Surgical cytoreduction for recurrent epithelial ovarian cancer. Cochrane Database Syst Rev 2013;(2):CD008765.

46. Du Bois A, Vergote I, Ferron G, et al. Randomized controlled phase III study evaluating the impact of secondary cytoreductive surgery in recurrent ovarian cancer: AGO DESKTOP III/ENGOT ov20. J Clin Oncol 2017;35:5501.

47. Coleman RL, Enserro D, Spirtos N, et al. A phase III randomized controlled trial of secondary surgical cytoreduction (SSC) followed by platinum-based combination chemotherapy $(\mathrm{PBC})$, with or without bevacizumab (B) in platinum-sensitive, recurrent ovarian cancer (PSOC): A NRG Oncology/Gynecologic Oncology Group (GOG) study. J Clin Oncol 2017;36:5501.

48. Sehouli J, Grabowski JP. Surgery for recurrent ovarian cancer: Options and limits. Best Pract Res Clin Obstet Gynaecol 2017;41:88-95.

49. Falcone F, Scambia G, Benedetti Panici P, et al. Tertiary cytoreductive surgery in recurrent epithelial ovarian cancer: A multicentre MITO retrospective study. Gynecol Oncol 2017;147:66-72.

50. Arvas M, Salihoglu Y, Sal V, et al. Tertiary Cytoreduction for Recurrent Epithelial Ovarian Cancer: a Multicenter Study in Turkey. Asian Pac J Cancer Prev 2016;17:1909-15.

51. Karam AK, Santillan A, Bristow RE, et al. Tertiary cytoreductive surgery in recurrent ovarian cancer: selection criteria and survival outcome. Gynecol Oncol 2007;104:377-80.

52. Fotopoulou C, Zang R, Gultekin M, et al. Value of tertiary cytoreductive surgery in epithelial ovarian cancer: an international multicenter evaluation. Ann Surg Oncol 2013;20:1348-54.

53. Fanfani F, Fagotti A, Ercoli A, et al. Is There a Role for Tertiary (TCR) and Quaternary (QCR) Cytoreduction in 
Recurrent Ovarian Cancer? Anticancer Res 2015;35:6951-5.

54. Shih KK, Chi DS, Barakat RR, et al. Beyond tertiary cytoreduction in patients with recurrent epithelial ovarian, fallopian tube, or primary peritoneal cancer. Gynecol Oncol 2010;116:364-9.

55. Cianci S, Abatini C, Fagotti A, et al. Hyperthermic intraperitoneal chemotherapy (HIPEC) for peritoneal malignancies using new hybrid CO2 system: preliminary experience in referral center. Updates Surg 2019;71:555-60.

56. Koole S, van Stein R, Sikorska K, et al. Primary cytoreductive surgery with or without hyperthermic intraperitoneal chemotherapy (HIPEC) for FIGO stage III epithelial ovarian cancer: OVHIPEC-2, a phase III randomized clinical trial. Int J Gynecol Cancer 2020. [Epub ahead of print].

57. Cianci S, Ronsini C, Vizzielli G, et al. Cytoreductive surgery followed by HIPEC repetition for secondary ovarian cancer recurrence. Updates Surg 2019;71:389-94.

58. Manzanedo I, Pereira F, Perez-Viejo E, et al.

Hyperthermic intraoperative intraperitoneal chemotherapy (HIPEC) with primary or secondary cytoreductive surgery in the treatment of advanced epithelial ovarian cancer. Minerva Ginecol 2017;69:119-27.

59. Spiliotis J, Halkia E, Lianos E, et al. Cytoreductive surgery and HIPEC in recurrent epithelial ovarian cancer: a prospective randomized phase III study. Ann Surg Oncol 2015;22:1570-5

60. Fagotti A, Costantini B, Gallotta V, et al. Minimally invasive secondary cytoreduction plus HIPEC versus open surgery plus HIPEC in isolated relapse from ovarian cancer: a retrospective cohort study on perioperative outcomes. J Minim Invasive Gynecol 2015;22:428-32.

61. Petrillo M, De Iaco P, Cianci S, et al. Long-Term Survival for Platinum-Sensitive Recurrent Ovarian Cancer Patients Treated with Secondary Cytoreductive Surgery Plus Hyperthermic Intraperitoneal Chemotherapy (HIPEC). Ann Surg Oncol 2016;23:1660-5.

62. Petrillo M, Zucchetti M, Cianci S, et al. Pharmacokinetics of cisplatin during open and minimally-invasive secondary cytoreductive surgery plus HIPEC in women with platinum-sensitive recurrent ovarian cancer: a prospective study. J Gynecol Oncol 2019;30:e59.

63. Monk BJ, Minion LE, Coleman RL. Anti-angiogenic agents in ovarian cancer: past, present, and future. Ann Oncol 2016;27 Suppl 1:i33-9.

64. Monk BJ, Han E, Josephs-Cowan CA, et al. Salvage bevacizumab (rhuMAB VEGF)-based therapy after multiple prior cytotoxic regimens in advanced refractory epithelial ovarian cancer. Gynecol Oncol 2006;102:140-4.

65. Burger RA, Sill MW, Monk BJ, et al. Phase II trial of bevacizumab in persistent or recurrent epithelial ovarian cancer or primary peritoneal cancer: a Gynecologic Oncology Group Study. J Clin Oncol 2007;25:5165-71.

66. Aghajanian C, Blank SV, Goff BA, et al. OCEANS: a randomized, double-blind, placebo-controlled phase III trial of chemotherapy with or without bevacizumab in patients with platinum-sensitive recurrent epithelial ovarian, primary peritoneal, or fallopian tube cancer. J Clin Oncol 2012;30:2039-45.

67. Burger RA, Brady MF, Bookman MA, et al. Incorporation of bevacizumab in the primary treatment of ovarian cancer. N Engl J Med 2011;365:2473-83.

68. Aravantinos G, Pectasides D. Bevacizumab in combination with chemotherapy for the treatment of advanced ovarian cancer: a systematic review. J Ovarian Res 2014;7:57.

69. Pignata S, Lorusso D, Joly F, et al. Chemotherapy plus or minus bevacizumab for platinum-sensitive ovarian cancer patients recurring after a bevacizumab containing first line treatment: the randomized phase 3 trial MITO16BMaNGO OV2B-ENGOT OV17. J Clin Oncol 2018;36:5506.

70. Ledermann JA, Embleton AC, Raja F, et al. Cediranib in patients with relapsed platinum-sensitive ovarian cancer (ICON6): a randomised, double-blind, placebo-controlled phase 3 trial. Lancet 2016;387:1066-74.

71. Matulonis UA, Berlin S, Ivy P, et al. Cediranib, an oral inhibitor of vascular endothelial growth factor receptor kinases, is an active drug in recurrent epithelial ovarian, fallopian tube, and peritoneal cancer. J Clin Oncol 2009;27:5601-6.

72. Barra F, Lagana AS, Ghezzi F, et al. Nintedanib for Advanced Epithelial Ovarian Cancer: A Change of Perspective? Summary of Evidence from a Systematic Review. Gynecol Obstet Invest 2019;84:107-17.

73. Monk BJ, Poveda A, Vergote I, et al. Final results of a phase 3 study of trebananib plus weekly paclitaxel in recurrent ovarian cancer (TRINOVA-1): Long-term survival, impact of ascites, and progression-free survival-2. Gynecol Oncol 2016;143:27-34.

74. Scott CL, Swisher EM, Kaufmann SH. Poly (ADPribose) polymerase inhibitors: recent advances and future development. J Clin Oncol 2015;33:1397-406.

75. Farmer H, McCabe N, Lord CJ, et al. Targeting the DNA repair defect in BRCA mutant cells as a therapeutic strategy. Nature 2005;434:917-21.

76. Network CGAR. Integrated genomic analyses of ovarian 
carcinoma. Nature 2011;474:609.

77. Pennington KP, Walsh T, Harrell MI, et al. Germline and somatic mutations in homologous recombination genes predict platinum response and survival in ovarian, fallopian tube, and peritoneal carcinomas. Clin Cancer Res 2014;20:764-75.

78. Konecny GE, Oza A, Tinker A, et al. Rucaparib in patients with relapsed, primary platinum-sensitive highgrade ovarian carcinoma with germline or somatic BRCA mutations: integrated summary of efficacy and safety from the phase II study ARIEL2. Gynecol Oncol 2017;145:2.

79. Ledermann JA, Harter P, Gourley C, et al. Overall survival in patients with platinum-sensitive recurrent serous ovarian cancer receiving olaparib maintenance monotherapy: an updated analysis from a randomised, placebo-controlled, double-blind, phase 2 trial. Lancet Oncol 2016;17:1579-89.

80. Pujade-Lauraine E, Ledermann JA, Selle F, et al. Olaparib tablets as maintenance therapy in patients with platinumsensitive, relapsed ovarian cancer and a BRCA1/2 mutation (SOLO2/ENGOT-Ov21): a double-blind, randomised, placebo-controlled, phase 3 trial. Lancet Oncol 2017;18:1274-84.

81. Kristeleit R, Shapiro GI, Burris HA, et al. A Phase I-II Study of the Oral PARP Inhibitor Rucaparib in Patients with Germline BRCA1/2-Mutated Ovarian Carcinoma or Other Solid Tumors. Clin Cancer Res 2017;23:4095-106.

82. Oza AM, Tinker AV, Oaknin A, et al. Antitumor activity and safety of the PARP inhibitor rucaparib in patients with high-grade ovarian carcinoma and a germline or somatic BRCA1 or BRCA2 mutation: Integrated analysis of data from Study 10 and ARIEL2. Gynecol Oncol 2017;147:267-75.

83. Swisher EM, Lin KK, Oza AM, et al. Rucaparib in relapsed, platinum-sensitive high-grade ovarian carcinoma (ARIEL2 Part 1): an international, multicentre, openlabel, phase 2 trial. Lancet Oncol 2017;18:75-87.

84. Coleman RL, Oza AM, Lorusso D, et al. Rucaparib maintenance treatment for recurrent ovarian carcinoma after response to platinum therapy (ARIEL3): a randomised, double-blind, placebo-controlled, phase 3 trial. Lancet 2017;390:1949-61.

85. Mirza MR, Monk BJ, Herrstedt J, et al. Niraparib Maintenance Therapy in Platinum-Sensitive, Recurrent Ovarian Cancer. N Engl J Med 2016;375:2154-64.

86. Ledermann J, Harter P, Gourley C, et al. Olaparib maintenance therapy in platinum-sensitive relapsed ovarian cancer. N Engl J Med 2012;366:1382-92.

87. Oza AM, Cibula D, Benzaquen AO, et al. Olaparib combined with chemotherapy for recurrent platinumsensitive ovarian cancer: a randomised phase 2 trial. Lancet Oncol 2015;16:87-97.

88. Oza AM, Matulonis UA, Malander S, et al. Quality of life in patients with recurrent ovarian cancer treated with niraparib versus placebo (ENGOT-OV16/NOVA): results from a double-blind, phase 3 , randomised controlled trial. Lancet Oncol 2018;19:1117-25.

89. Friedlander M, Gebski V, Gibbs E, et al. Health-related quality of life and patient-centred outcomes with olaparib maintenance after chemotherapy in patients with platinumsensitive, relapsed ovarian cancer and a BRCA1/2 mutation (SOLO2/ENGOT Ov-21): a placebo-controlled, phase 3 randomised trial. Lancet Oncol 2018;19:1126-34.

90. Gunderson CC, Matulonis U, Moore KN. Management of the toxicities of common targeted therapeutics for gynecologic cancers. Gynecol Oncol 2018;148:591-600.

91. Oronsky B, Ray CM, Spira AI, et al. A brief review of the management of platinum-resistant-platinum-refractory ovarian cancer. Med Oncol 2017;34:103.

92. Gynecologic Oncology G, Markman M, Blessing J, et al. Phase II trial of weekly paclitaxel $(80 \mathrm{mg} / \mathrm{m} 2)$ in platinum and paclitaxel-resistant ovarian and primary peritoneal cancers: a Gynecologic Oncology Group study. Gynecol Oncol 2006;101:436-40.

93. Rose PG, Blessing JA, Ball HG, et al. A phase II study of docetaxel in paclitaxel-resistant ovarian and peritoneal carcinoma: a Gynecologic Oncology Group study. Gynecol Oncol 2003;88:130-5.

94. Rose PG, Blessing JA, Mayer AR, et al. Prolonged oral etoposide as second-line therapy for platinum-resistant and platinum-sensitive ovarian carcinoma: a Gynecologic Oncology Group study. J Clin Oncol 1998;16:405-10.

95. Mutch DG, Orlando M, Goss T, et al. Randomized phase III trial of gemcitabine compared with pegylated liposomal doxorubicin in patients with platinum-resistant ovarian cancer. J Clin Oncol 2007;25:2811-8.

96. Ferrandina G, Ludovisi M, Lorusso D, et al. Phase III trial of gemcitabine compared with pegylated liposomal doxorubicin in progressive or recurrent ovarian cancer. J Clin Oncol 2008;26:890-6.

97. Gordon AN, Tonda M, Sun S, et al. Long-term survival advantage for women treated with pegylated liposomal doxorubicin compared with topotecan in a phase 3 randomized study of recurrent and refractory epithelial ovarian cancer. Gynecol Oncol 2004;95:1-8.

98. Sehouli J, Stengel D, Harter P, et al. Topotecan Weekly Versus Conventional 5-Day Schedule in Patients With 
Platinum-Resistant Ovarian Cancer: a randomized multicenter phase II trial of the North-Eastern German Society of Gynecological Oncology Ovarian Cancer Study Group. J Clin Oncol 2011;29:242-8.

99. Cannistra SA, Matulonis UA, Penson RT, et al. Phase II study of bevacizumab in patients with platinum-resistant ovarian cancer or peritoneal serous cancer. J Clin Oncol 2007;25:5180-6.

100.Pujade-Lauraine E, Hilpert F, Weber B, et al. AURELIA: A randomized phase III trial evaluating bevacizumab combined with chemotherapy for platinum-resistant recurrent ovarian cancer. J Clin Oncol 2012;30:327s.

101.Pujade-Lauraine E, Hilpert F, Weber B, et al. Bevacizumab combined with chemotherapy for platinum-resistant recurrent ovarian cancer: The AURELIA open-label randomized phase III trial. J Clin Oncol 2014;32:1302-8.

102. Stockler MR, Hilpert F, Friedlander M, et al. Patientreported outcome results from the open-label phase III AURELIA trial evaluating bevacizumab-containing therapy for platinum-resistant ovarian cancer. J Clin Oncol 2014;32:1309-16.

103. Musa F, Pothuri B, Blank SV, et al. Phase II study of irinotecan in combination with bevacizumab in recurrent ovarian cancer. Gynecol Oncol 2017;144:279-84.

Cite this article as: Garzon S, Laganà AS, Casarin J, Raffaelli R, Cromi A, Franchi M, Barra F, Alkatout I, Ferrero S, Ghezzi F. Secondary and tertiary ovarian cancer recurrence: what is the best management? Gland Surg 2020;9(4):1118-1129. doi: 10.21037/ gs-20-325
104. Nagao S, Kogiku A, Suzuki K, et al. A phase II study of the combination chemotherapy of bevacizumab and gemcitabine in women with platinum-resistant recurrent epithelial ovarian, primary peritoneal, or fallopian tube cancer. J Ovarian Res 2020;13:14.

105.Shoji T, Komiyama S, Kigawa J, et al. An open-label, randomized, phase II trial evaluating the efficacy and safety of standard of care with or without bevacizumab in platinum-resistant epithelial ovarian, fallopian tube, or primary peritoneal cancer patients previously treated with bevacizumab for front-line or platinum-sensitive ovarian cancer: rationale, design, and methods of the Japanese Gynecologic Oncology Group study JGOG3023. BMC Cancer 2018;18:771.

106.Kaufman B, Shapira-Frommer R, Schmutzler RK, et al. Olaparib monotherapy in patients with advanced cancer and a germ-line BRCA1/2 mutation: An open-label phase II study. J Clin Oncol 2013;31:11024.

107. Matulonis UA, Penson RT, Domchek SM, et al. Olaparib monotherapy in patients with advanced relapsed ovarian cancer and germline BRCA1/2 mutation: a multistudy analysis of response rates and safety. Ann Oncol 2016;27:1013-9. 